\title{
Is Operative Diagnosis for Revision Total Hip Arthroplasty Related to Patient Reported Outcomes?
}

\author{
Erik Holder BA BS ${ }^{1}$, Alex Ciesielski BS ${ }^{1}$, \\ Mary Ziemba-Davis $\mathrm{BA}^{2}$, R. Michael Meneghini $\mathrm{MD}^{1,2}$ \\ ${ }^{1}$ Indiana University School of Medicine, Indianapolis, IN \\ ${ }^{2}$ IU Health Physicians, Orthopedics \& Sports Medicine, Fishers, IN
}

Background and Hypothesis: Component loosening and instability are the leading causes of revision total hip arthroplasty (THA). The purpose of this study was to compare patient-reported outcomes after revision THA based on failure etiology. We hypothesized that outcomes would differ based on reason for revision.

Project Methods: 187 consecutive revision THAs performed between 2010 and 2017 were retrospectively reviewed. Prospectively collected preoperative and minimum one-year Hip Disability and Osteoarthritis Outcome Score/HOOS Jr., UCLA Activity Level, WOMAC Index, and patient satisfaction were assessed based on failure etiology. Demographic variables and covariates were accounted for including sex, age, BMI, ASA classification, heart disease, lumbar spine pathology, narcotic use, fibromyalgia, depression, and autoimmune arthritis.

Results: Latest UCLA activity level did not differ based on failure etiology $(p=0.381)$. However, the degree of improvement in activity level was higher $(p=$ $0.04)$ in patients revised for loosening, instability, and infection compared to ALTR and polyethylene wear. HOOS Jr $(p=0.949)$ and WOMAC total $(p=0.147)$ scores did not differ based on failure etiology at latest follow-up, although patients revised for loosening had greater WOMAC improvement compared to all other groups except polyethylene wear $(p=0.016)$. Satisfaction did not vary based on failure etiology $(p=0.365)$, and demographic and covariates were unrelated to outcomes $(p \geq 0.165)$.

Conclusion and Potential Impact: We observed that patient-reported outcomes following revision THA vary based on revision reason and activity level improvement is mitigated patients revised for ALTR and poly wear. These findings may help surgeons and patients alike set expectations for recovery following revision THA. 